\title{
Integración de la Docencia y la Extensión en el aula de Química
}

\author{
Integration of Teaching and Extension in the Chemistry classroom
}

\section{Sofía Sampaolesi}

https://orcid.org/0000-0001-8080-9181

sampaolesi@quimica.unlp.edu.ar

Facultad de Ciencias Exactas | UNLP | Argentina
Silvina Mariela Justianovich

https://orcid.org/0000-0002-5802-8853

sjustianovich@gmail.com

UNLP | Argentina

\author{
Laura Estefanía Briand \\ https://orcid.org/0000-0002-6828-8624 \\ briand@quimica.unlp.edu.ar \\ Facultad de Ciencias Exactas | UNLP | Argentina
}

\section{RESUMEN}

Reconociendo la importancia de los aportes de la Extensión a la formación de profesionales comprometidos con su rol social y de su potencial para otorgar significatividad a los contenidos disciplinares del currículum de las carreras de grado, trayendo un contexto social que les dé sentido y habilite su representación, el presente artículo aborda reflexiones acerca de la necesidad de construir nuevos lazos entre los aportes y propuestas de la Extensión desarrollada en la Facultad de Ciencias Exactas y las propuestas de enseñanza. En este marco, se da cuenta de los posicionamientos docentes que sustentaron el diseño de una serie de trabajos prácticos de laboratorio de la asignatura Introducción a la Química. Estos trabajos de laboratorio pretenden motivar el aprendizaje de la Química a través de la búsqueda de posibles soluciones a problemas concretos de la comunidad inmediata, fomentando una actitud proactiva de las/os estudiantes en la elaboración de nuevos saberes en y a través de la práctica. La construcción metodológica puso el eje en propiciar situaciones que promuevan aprendizajes significativos trayendo la Extensión al aula universitaria.

\section{SUMMARY}

Recognizing the meaningful contributions of university Extension to the training of professionals committed to their social role and its potential to give significance to the curricular disciplinary contents, bringing a social context that gives them meaning and enables their representation, this paper addresses thoughts about the need to build new bonds between the contributions and proposals of the Extension activities developed in the Faculty of Exact Sciences and the teaching proposals. In this framework, the teaching positions that supported the design of a series of practical laboratory assignments of the subject Introduction to Chemistry are realized. These laboratory assignments are intended to motivate the learning of Chemistry through the search for possible solutions to specific problems of the nearby community, encouraging a proactive attitude of the students in the elaboration of new knowledge in and through practice. The methodological construction focuses on fostering situations that promote meaningful learning by bringing Extension to the university classroom.

\author{
PALABRAS CLAVE \\ docencia, \\ extensión universitaria, \\ integralidad de funciones \\ en el aula, \\ aprendizaje significativo \\ contextualizado.
}

\section{KEY WORDS}

University teaching, university Extension, integration of university functions in the classroom, contextualized meaningful learning. 
El presente texto aborda las reflexiones y problematizaciones suscitadas durante el quehacer de un equipo docente de la cátedra de Introducción a la Química y Química General (IQQG), a cargo de las asignaturas homónimas Introducción a la Química (IQ) y Química General (QG), cuáles forman parte del primer año del plan de estudios de un conjunto de carreras de grado1 de la Facultad de Ciencias Exactas de la UNLP (FCE-UNLP). Estas nacieron de observaciones compartidas por las/os docentes, vinculadas a dificultades para motivar el aprendizaje de la Química y lograr aprendizajes más significativos y menos mecanísticos, y encontraron potenciales respuestas en la integración virtuosa de los ámbitos de la enseñanza y el aprendizaje y de la extensión universitaria, que se constituyó en oportunidad para el diseño de una propuesta innovadora de los Trabajos Prácticos de laboratorio para la asignatura IQ, en el marco del Curso con Estrategias Alternativas para la Enseñanza de la Química (CEAEQ) (Barraqué y col., en prensa).

\section{APORTES DE LA EXTENSIÓN A LA FORMACIÓN DE PROFESIONALES}

La Extensión universitaria es un concepto profundamente polisémico e históricamente determinado, re-elaborado y re-significado por las y los actores sociales de cada contexto, signado por sus trayectorias de vida. El presente trabajo recupera la concepción de Extensión crítica, definida por Tommasino y Cano como aquella que:

(...) tiene dos objetivos centrales: uno vinculado con la formación de los universitarios y la posibilidad de establecer procesos integrales que rompan con la formación profesionalista alejada de un criterio de compromiso social de los graduados universitarios (...) y procurar procesos formativos integrales que generen universitarios solidarios y comprometidos con los procesos de transformación de la sociedad. Un segundo objetivo se propone contribuir a los procesos de organización y autonomía de los sectores populares subalternos, intentando contribuir a la generación de poder popular. En una perspectiva de cambio universitario y social, estos dos objetivos tienen una vinculación orgánica: no es posible avanzar en uno sin avanzar en el otro (Tommasino y Cano, 2016: 10). 
Desde esta perspectiva, la Extensión tiene un rol fundamental en la formación de universitarias y universitarios que se identifiquen como actores sociales fundamentales del cambio social dirigido a empoderar a los sectores postergados de su comunidad inmediata, conscientes de que, gracias a la Universidad pública, pueden poner al servicio de este cambio conocimientos y herramientas a las que otras/os ciudadanas/ os no tuvieron acceso. Profundizando en estos sentidos, Cano Menoni propone a la extensión crítica como:

(...) un medio idóneo para la formación de los estudiantes, ya no desde el punto de vista del entrenamiento profesional, sino desde una doble motivación: a) el potencial formativo de la experiencia (desde las tradiciones de autores como Dewey y Decroly); y b) el potencial de la extensión para la formación humanista y crítica de los estudiantes (desde la tradición universitaria anti-utilitarista de corte rodoniano) (Cano Menoni, 2014: 238).

Discusiones respecto del perfil de los profesionales que se forman en nuestra casa de estudios se suscitan con frecuencia en el ámbito de la Extensión, nacidas en el propio quehacer extensionista y fomentadas entre las/os participantes de los proyectos. La interpelación que el trabajo en territorio y el vínculo con sus actrices y actores generan en las/os estudiantes, les llevan a concienciar problemas sociales concretos cuya solución puede abordarse a través de los conocimientos adquiridos durante su trayectoria académica, y les propone el establecimiento de vínculos de colaboración desde los cuales buscar esas soluciones. Esta mirada de la propia formación suele ser novedosa para quienes se inician en la extensión; se manifiesta allí la singularidad o vacancia de propuestas curriculares que hagan eje, o siquiera expliciten, la aplicabilidad de los conocimientos académicos en contextos y territorios concretos.

Pese a los intentos de convocar ampliamente a toda la comunidad de la FCE a estos debates, la realidad indica que no logran trascender el ámbito de la Extensión. El actual perfil del egresado de nuestra casa de estudios es el de un profesional capacitado para la investigación científica tanto básica como aplicada; para desenvolverse en el ámbito industrial en las áreas de investigación y desarrollo (I+D), producción y control de calidad; y para desenvolverse en el área de Salud, únicamente los títulos habilitantes de las carreras de Farmacia y Licenciatura en Bioquímica. Estos perfiles están definidos a través de las incumbencias del título y sus ámbitos de desempeño; no se encuentran publicados documentos que reflejen un debate acerca del rol social de los profesionales que se forman en la FCE, como tampoco documentos que den discusión acerca de la concepción de Ciencia que sostiene el currículum de sus carreras 
y las prácticas docentes arraigadas.

Este adeudado debate dificulta aún más el lograr consenso acerca de lo que se quiere enseñar y de cómo hacerlo hacia el interior de las cátedras. Como equipo docente con trayectoria de participación en proyectos y programas de Extensión y de gestión en la Secretaría de Extensión de la FCE, reconocemos los aportes de la Extensión a las discusiones en torno a la formación de profesionales de excelencia comprometidas/os con su rol social y a los debates acerca de por qué se enseña lo que se enseña en la facultad y de cómo se enseña lo que se enseña dentro de la cátedra.

\section{Como equipo docente con trayectoria de participación en proyectos y programas de Extensión y de gestión en la Se- cretaría de Extensión de la FCE, reconocemos los aportes de la Extensión a las discusiones en torno a la formación de profesionales de excelencia comprometidas/os con su rol social y a los debates acerca de por qué se enseña lo que se enseña en la facultad y de cómo se enseña lo que se enseña dentro de la cátedra.}

Desde otra mirada, el potencial formativo de la experiencia asociado a la extensión crítica, como lo describe Cano Menoni (2014: 238), se nos presenta como oportunidad a través de la cual generar condiciones para un aprendizaje contextualizado y significativo de la disciplina científica, trayendo desde el territorio aquellas problemáticas plausibles de ser abordadas desde los conocimientos que una/un estudiante elabora en las clases de química de primer año. Esta idea no nace en la intención de sustituir la metodología de clase tradicional de la cátedra, sino de brindar un enfoque complementario, en el que la Extensión aporta un marco contextual que viabiliza el aprendizaje de la disciplina discutida en el aula a través de significantes y representaciones concretas de la realidad local inmediata.

\section{¿EXTENSIÓN EN EL AULA O DOCENCIA EN EL TERRITORIO?}

A partir de estos debates, nos propusimos diseñar una serie de trabajos prácticos de laboratorio que abordasen la enseñanza y el aprendizaje de los conceptos disciplinares establecidos en el programa de la asignatura IQQG a través de una práctica experimental nacida en respuesta a una demanda de la comunidad local. En esta instancia, se llevó adelante un trabajo coordinado con miembros del proyecto de extensión Taller de Aguas, que funciona dentro de la FCE. El Taller de 
Potabilidad de Aguas trabaja en pos de defender el derecho al agua potable de la población más vulnerable, como elemento fundamental para garantizar la salud pública, a través de la toma de muestras de agua de consumo, su análisis en el laboratorio, la devolución de los resultados y la construcción colectiva de soluciones con la comunidad. La realización de la etapa fisicoquímica del análisis de las muestras de agua traídas del territorio durante los trabajos prácticos de laboratorio de IQ se presentó como una oportunidad de integración de la extensión y la docencia en el aula, a través de la cual otorgar significado y relevancia a los contenidos de química que se pretende sean aprendidos por las/os estudiantes.

Nos ocupó especialmente la idea de "traer la Extensión" y las demandas del territorio al aula, como contrapunto de propuestas de articulación que plantean salidas a campo, llevando la docencia al territorio. A continuación, daremos cuenta de las reflexiones pedagógicas y como extensionistas que nos llevan a abogar por este enfoque de integración de las funciones universitarias.

\section{Nos ocupó especialmente la idea de "traer la Extensión" y las demandas del territorio al aula, como contrapunto de pro- puestas de articulación que plantean salidas a campo, llevan- do la docencia al territorio.}

Entendemos que la Extensión como articuladora del territorio y el aula fomenta la pregunta investigativa y una actitud pro-activa de la/ el estudiante, que se ve motivada/o en la búsqueda de respuestas y soluciones a la problemática planteada por las/os actores del territorio. En este sentido, no sólo puede aportar a motivar a las/os estudiantes en el aprendizaje de la Química como herramienta de cambio social, sino que también puede disputar la idea tradicional del saber académico que producen otras/os, que está en los libros, que"se tiene" o "se adquiere". En el contexto de una práctica de laboratorio surgida en respuesta a una demanda concreta, las soluciones y los saberes se construyen, se elaboran en un hacer aplicado a la singularidad de la situación planteada. Una propuesta educativa de estas características se reviste de las potencialidades de un practicum, en los términos en que lo describió Schön (1993, en Morandi, 1997).

En muchas ocasiones, de acuerdo a nuestra experiencia docente, la teoría y la práctica se presentan para las/os estudiantes de primer año como mundos paralelos dentro de una misma asignatura. En este sentido, la adopción de un practicum como diseño metodológico habilita al trabajo práctico de laboratorio como un ámbito de aprendizaje más y evita reducirlo a la mera aplicación de soluciones 
predeterminadas discutidas en las "clases teóricas", dando debate a la falsa dicotomía teoría-práctica. Por otro lado, entendemos que diseños metodológicos que articulan las funciones universitarias de forma secuencial a través de una planificación de los momentos de la "práctica", llevada a cabo durante actividades en el territorio en el marco de un proyecto de Extensión, disociada y posterior a la "teoría", que ocurre en el aula, podrían reforzar nociones previas de fragmentación entre la teoría y la práctica.

Del lado de la Extensión, la articulación de las funciones universitarias en el ámbito del aula, entorno legítimo de la enseñanza y el aprendizaje avalado por siglos de tradición académica, puede redundar en su jerarquización, aunándola en un mismo espacio y tiempo con la función más reconocida de la Universidad. Es también nuestra expectativa que el contacto con actividades extensionistas desde el primer año de su carrera promueva la participación de más estudiantes y más docentes en proyectos y programas de Extensión.

\section{Del lado de la Extensión, la articulación de las funciones universitarias en el ámbito del aula, entorno legítimo de la enseñanza y el aprendizaje avalado por siglos de tradición académica, puede redundar en su jerarquización, aunándo- la en un mismo espacio y tiempo con la función más recono- cida de la Universidad.}

Retomando la concepción de extensión crítica como aquella que interpela a las/os estudiantes y promueve debates acerca de su propia formación, de su rol como futuras/os profesionales en su comunidad y de la Ciencia como herramienta transformadora de la realidad social, entendemos que llevar la Extensión al aula garantizará que estas discusiones formen parte del currículum y sean abordadas de forma masiva por la comunidad de Exactas, evitando que queden circunscritas a ámbitos reducidos de participación voluntaria, como son las actividades extensionistas, y/o que cristalicen en prácticas aisladas dependientes de la voluntad de debate de la/el docente de ocasión.

\section{DESARROLLO METODOLÓGICO}

La decisión como equipo docente de permeabilizar el aula a lo que ocurre fuera de ella tiene explicación, no sólo en los vínculos entre los elementos de la situación educativa que estimamos fomentar, sino también en nuestra trayectoria como educadores y extensionistas: "La adopción por parte del docente de una perspectiva axiológica, ideológica (...), incide en las formas de vinculación con el conocimiento cuya 
interiorización se propone, y por lo tanto, también tiene su expresión en la construcción metodológica" (Edelstein, 1995: 85).

El diseño de los trabajos prácticos de laboratorio que integran la Extensión al aula se nutrió de los intercambios entre las/os docentes del equipo de IQ y las/os extensionistas del Taller de Aguas. Se trata de una experiencia de curricularización de la extensión cuyo objetivo es aportar a una perspectiva constructivista de la enseñanza-aprendizaje de la Química. Desde esta posición, se asume que curricularizar la extensión en el aula, a través de los tiempos y las actividades áulicas, es una modalidad que aborda ciertas problemáticas que la curricularización a través de la territorialización de la enseñanza y el aprendizaje no habilita, como se discutió en párrafos previos.

Desde esta posición, se asume que curricularizar la extensión en el aula, a través de los tiempos y las actividades áulicas, es una modalidad que aborda ciertas problemáticas que la curricularización a través de la territorialización de la enseñanza y el aprendizaje no habilita, como se discutió en párrafos previos.

Edelstein sostiene el diseño de las prácticas de enseñanza dentro de la construcción metodológica que realiza el docente como "...fruto de un acto singularmente creativo de articulación entre la lógica disciplinar, las posibilidades de apropiación de ésta por parte de los sujetos, y las situaciones y los contextos particulares que constituyen ámbitos donde ambas lógicas se entrecruzan" (Edelstein, 1995: 85). Como equipo docente consideramos que el contexto traído desde el territorio a través de las demandas de sus actrices y actores habilita la articulación de la teoría y la práctica en el aula, el aprendizaje de la disciplina en su aplicación, y motiva la construcción de saberes a través de la búsqueda de respuestas a esas demandas.

Más aún, no sólo promueve un interés por el aprendizaje de la Química, sino que requiere de la/el estudiante una actitud proactiva, propositiva y comprometida con su propio aprendizaje, abandonando actitudes pasivas frente al estudio, muchas veces adoptadas durante la trayectoria escolar previa. La autonomía como estudiantes y el rol activo que buscamos promover, consideramos son fundamentales para completar su formación de grado y en el ejercicio como futuras/ os profesionales. Por otro lado, la actividad en el laboratorio buscará estimular el trabajo en grupos y la construcción colectiva de saberes, acercando ciertas dimensiones de la ecología de saberes. Se estima que la problemática social traída al aula tiene potencial como disparador para que las/os estudiantes compartan saberes de su tradición 
familiar, saberes artísticos y populares y los pongan en convivencia con los saberes científicos que circulan en el ambiente académico, acercándose a concepciones más holísticas del saber, resignificando y abonando, también desde la memoria emotiva, al logro de aprendizajes significativos.

\section{CONCLUSIONES}

El diseño de estos trabajos prácticos habilitó un intercambio en el que se gestó un equipo de educadoras/es, conformado por docentes y extensionistas de la FCE, que se nutre de las perspectivas traídas desde cada campo, contribuyendo a la integralidad de las funciones universitarias de Docencia y Extensión. Asimismo, este vínculo entre docentes y extensionistas sienta las bases para la formación de futuros equipos inter-disciplinarios (inter-funciones universitarias) desde el diálogo entre pares, donde puedan ser ponderados todos los aportes, sin primacía de funciones ni de jerarquías de cargo.

Llevar adelante la elaboración de la propuesta permitió identificar aspectos de la fragmentación de la Docencia y la Extensión (también la Investigación) en el currículum de las carreras de la FCE, artefacto disociativo de la práctica académica que obtura oportunidades de enriquecer los procesos de enseñanza y aprendizaje en la formación de profesionales científicos. En el caso particular de la Extensión, su ausencia en los discursos y actividades en el aula tiene como consecuencia adicional que la mayoría de las/os estudiantes la desconozca como función pilar de la Universidad, dificultando la difusión y convocatoria a actividades extensionistas extracurriculares. Como producto de la discusión y promoción de la Extensión en el aula, se espera una mayor participación estudiantil y docente en los programas y proyectos de la FCE.

En tanto toda propuesta pedagógica es política, no podría pensarse la enseñanza sin sujetos, sin una intencionalidad que la oriente, ni bajo la premisa de la mera aplicación de una serie de contenidos teóricos a problemas reales que se encuentran fuera de las aulas universitarias. Desde el posicionamiento asumido en el diseño metodológico de estos trabajos prácticos de laboratorio, se apostó a que la contextualización de la enseñanza generará mejores condiciones para habilitar procesos de aprendizaje significativos y un compromiso con las problemáticas sociales de la comunidad en la que las/os futuras/os profesionales se desempeñarán. Estimamos que los debates suscitados a este respecto desde el primer año de carrera promoverán una impronta de formación de profesionales al servicio de las causas sociales transversal a la formación de excelencia disciplinar, académica y científica que caracteriza a la FCE-UNLP. Asimismo, la implementación de propuestas de enseñanza que contextualicen las disciplinas a través de una práctica profesional real son singulares e innovadoras en el primer año de carrera, particu- 
larmente en el contexto de nuestra casa de estudios.

En este sentido, vemos necesario retomar los debates acerca del perfil de las/os profesionales científicas/os que forma la universidad pública y rebatir aquellas nociones que instalan que el compromiso social y la excelencia académica son formas alternativas, mutuamente excluyentes y opcionales de vivir la profesión. Como expectativa última, esperamos que propuestas educativas como ésta inicien discusiones que involucren a toda la comunidad facultativa acerca de aspectos tan fundamentales y transversales a la institución como son el perfil de los profesionales que forma y la valoración de la Extensión como paradigma y espacio formativo de profesionales de calidad socialmente comprometidos.

\section{Notas:}

Licenciatura en Química, Licenciatura en Química y Tecnología Ambiental, Licenciatura en Bioquímica, Farmacia, Licenciatura en Ciencia y Tecnología de Alimentos, Licenciatura en Óptica Ocular y Optometría, Licenciatura en Biotecnología y Biología Molecular, Tecnicatura Universitaria en Alimentos y Tecnicatura Universitaria en Química. 


\section{BIBLIOGRAFIA}

Barraqué, F., Sampaolesi, S., Briand, L. E., Vetere, V. (En prensa) "Innovación metodológica para la enseñanza de la química durante el primer año de la universidad". Educación Química, 32(1).

Cano Menoni, A. (2014). "Extensión universitaria y alternativas pedagógicas en la Universidad Latinoamericana". Rodríguez A. B. y Contreras Montellanos O. F. (coord.). La construcción del futuro: los retos de las Ciencias Sociales en México Memorias del $4^{\circ}$ Congreso Nacional de Ciencias Sociales. 232-244. México: Cesmeca-Unicach.

Edelstein, G. (1995). “Lo metodológico: un capítulo pendiente en el debate didáctico". Corrientes Didácticas Contemporáneas. Buenos Aires: Editorial Paidós.

Morandi, G. (1997). “La relación teoría-práctica en la formación de profesionales: problemas y perspectivas". Ponencia presentada en las II Jornadas de Actualización en Odontología, Universidad Nacional de La Plata. La Plata, Argentina.

Tommasino, H. y Cano, A. (2016). "Avances y retrocesos de la extensión crítica en la Universidad de la República de Uruguay". Revista Masquedós, 1(1), 9-23. Tandil, Argentina: Secretaría de Extensión UNICEN.

http://ojs.extension.unicen.edu.ar/index.php/masquedos/article/view/3/2 